\title{
Neutron Scattering Studies on Magnetic Structure of the Double-Layered Manganite $\mathrm{La}_{2-2 x} \mathrm{Sr}_{1+2 x} \mathrm{Mn}_{2} \mathrm{O}_{7}(0.30 \leq x \leq 0.50)$
}

\author{
M. Kubota,${ }^{\dagger}$ H. Fujioka,${ }^{\ddagger}$ K. Ohoyama ${ }^{3}$ K. Hirota,${ }^{\ddagger}$ Y. Moritomo,${ }^{4}$ H. Yoshizawa,${ }^{\dagger}$ and Y. Endoh ${ }^{\ddagger}$ \\ ${ }^{\dagger}$ Neutron Scattering Laboratory, I.S.S.P., University of Tokyo, Tokai, Ibaraki, 319-1106, Japan \\ ${ }^{\ddagger}$ CREST, Department of Physics, Tohoku University, Aoba-ku, Sendai, 980-8578, Japan \\ ${ }^{3}$ Institute for Materials Research Tohoku University, Aoba-ku, Sendai 980-8577, Japan \\ ${ }^{4}$ Center for Integrated Research in Science and Engineering, Nagoya University, Nagoya, 464-01, Japan
}

\begin{abstract}
Systematic powder diffraction studies have been carried out to establish the magnetic phase diagram of $\mathrm{La}_{2-2 x} \mathrm{Sr}_{1+2 x} \mathrm{Mn}_{2} \mathrm{O}_{7}$ (LSMO327) in a wide hall concentration range $(0.30 \leq x \leq 0.50$ ), using the HERMES diffractometer. LSMO327 exhibits a planar ferromagnetic structure for $0.32 \leq x \leq 0.38$ at low temperatures. A finite canting angle between planar magnetic moments on neighboring planes starts appearing around $x \sim 0.40$ and reaches $180^{\circ}$ (A-type antiferromagnet) at $x=0.48$. At $x=0.30$, on the other hand, the magnetic moments are aligned parallel to the $c$-axis.
\end{abstract}

Keywords: A: magnetic materials, B: crystal growth, C: neutron scattering, D: magnetic structure

Perovskite $\mathrm{Mn}$ oxide $\mathrm{R}_{1-x} \mathrm{~A}_{x} \mathrm{MnO}_{3}$ (R: Rare-earth ion, A: Alkaline-earth element) provides an ideal stage to systematically study complex physics resulting from spincharge-lattice degrees of freedom. [1,2] Recently, Moritomo et al. [3, [4 have discovered that the layered Perovskite Mn oxide $\mathrm{La}_{2-2 x} \mathrm{Sr}_{1+2 x} \mathrm{Mn}_{2} \mathrm{O}_{7}$ (LSMO327) with $x=0.40$ shows colossal magnetoresistance (CMR), which is much more enhanced than that of similarly doped $\mathrm{La}_{1-x} \mathrm{Sr}_{x} \mathrm{MnO}_{3}$. LSMO327 (I/4mmm, $a=3.871 \AA$ and $c=20.126 \AA$. [5, 6]) has $\mathrm{MnO}_{2}$ double layers separated by $\left(\mathrm{La}_{1-x} \mathrm{Sr}_{x}\right)_{2} \mathrm{O}_{2}$, stacking along the $c$-axis.

The magnetic structure of LSMO327 has been established between $x=0.40$ and 0.48 by neutron-diffraction study using single crystals. [6] The low-temperature magnetic phase consists of planar ferromagnetic (FM) and Atype antiferromagnetic (AFM) components, indicating a canted AFM ordering. With increasing $x$, the canting angle between planes changes from $6.3^{\circ}$ at $x=0.40$ to $180^{\circ}$ at $x=0.48$, while the FM ordering temperature $T_{C}$ decreases from $120 \mathrm{~K}$ to $0 \mathrm{~K}$. It was also discovered that the A-type AFM ordering remains above $T_{C}$ up to $T_{N} \sim 200 \mathrm{~K}$. At $x=0.30$, on the other hand, it is reported that the low-temperature magnetic structure is AFM with the easy axis parallel to the $c$-axis and that the easy-axis shows a canting at higher temperature, giving the in-plane components. [7,8] As for $x=0.50$, superlattice peaks are observed by electron diffraction [9] as well as neutron diffraction, [10] which are ascribed to chargeordering. The A-type AFM structure is realized at low temperatures, while additional magnetic reflections are seen at intermediate temperatures, indicating the coexistence of CE-type and A-type AFM structures.

LSMO327 exhibits a variety of magnetic structures with changing temperature and the hole concentration $x$. There have been, however, no systematic study of the magnetism of LSMO327 in a wide temperature and hole concentration range so far. To establish the magnetic phase diagram, we have carried out extensive neutrondiffraction studies on LSMO327 powder samples prepared in consistent manner.
The prescribed amount of dried $\mathrm{La}_{2} \mathrm{O}_{3}, \mathrm{SrCO}_{3}$, and $\mathrm{Mn}_{3} \mathrm{O}_{4}$ are thoroughly mixed and calcined in the air at $1200-1450^{\circ} \mathrm{C}$ for 4 days with frequent grinding. Sample rods were melt-grown in a floating zone optical image furnace, then powderized again. All the samples were characterized by x-ray diffractions, which show no detectable impurities.

We have taken powder diffraction patterns at room temperature, intermediate temperature $(100-120 \mathrm{~K})$, and low temperature $(\sim 10 \mathrm{~K})$ using HERMES, 11] which is a powder neutron diffractometer with multidetectors with the Ge $\left(\begin{array}{lll}3 & 3 & 1\end{array}\right)$ monochromater $(\lambda=$ $1.819 \AA$ ). Temperature dependences of magnetic reflections were measured using the triple-axis spectrometers GPTAS and TOPAN, where the $\left(\begin{array}{lll}0 & 0 & 2\end{array}\right)$ reflection of pyrolytic graphite (PG) was used to monochromate, together with PG filters to eliminate higher order contaminations. These spectrometers are located in the JRR-3M reactor in JAERI.

Figure 1 shows five possible models of magnetic structure and corresponding scattering patterns for LSMO327. Here we assume that spins are ferromagnetically coupled within a plane, i.e., FM or A-type AFM phase. Provided that spins are antiferromagnetically modulated within the $a b$ plane, there should be magnetic superlattice peaks at ( $h k l$ ) with half integer of $h$ and/or $k$, which is clearly not the case for the present study. Models a (FM-I) and b (FM-II) correspond to FM structures with spins aligning within the $a b$ plane and along the $c$-axis, respectively. Models c (AMF-I), d (AFM-II) and e (AFM-III) represent A-type AFM structures with different alternating pattern along the $c$-axis. Note that a canted AFM magnetic structure is represented by a combination of FM and AFM components, which thus gives a combination of FM and AFM reflection patters shown in Fig. 1. 


\section{$\mathrm{La}_{2-2 x} \mathrm{Sr}_{1+2 x} \mathrm{Mn}_{2} \mathrm{O}_{7}$}

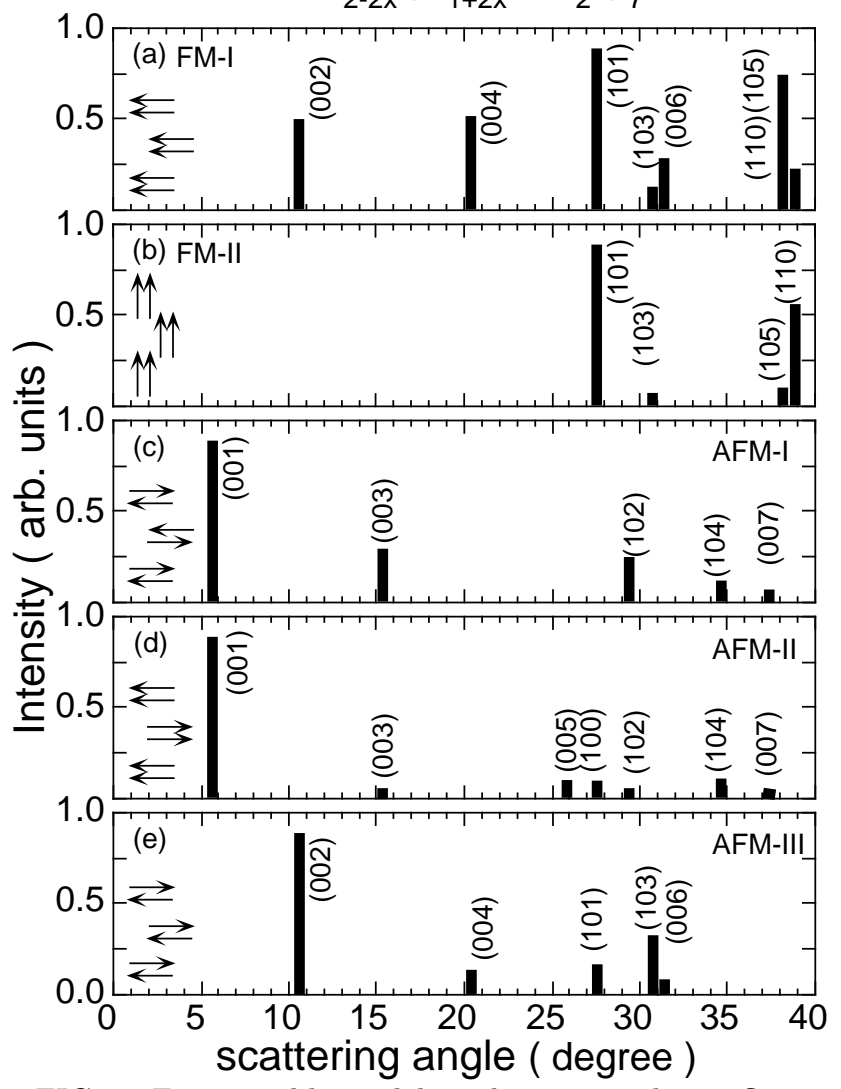

FIG. 1. Five possible models and corresponding reflections for magnetic structures which would explain the present results of $\mathrm{La}_{2-2 x} \mathrm{Sr}_{1+2 x} \mathrm{Mn}_{2} \mathrm{O}_{7}(0.30 \leq x \leq 0.50)$.

We have carried out comprehensive powder neutrondiffraction measurements at $x=0.30,0.32,0.335,0.35$, $0.38,0.39,0.40,0.45,0.48$ and 0.50 . Figure 2 summarizes the results for $x=0.30,0.35,0.39,0.45$ and 0.50 at $10 \mathrm{~K}$. We have noticed that the powder diffraction patterns for $x=0.32 \leq x \leq 0.50$ at low temperatures can be explained by a combination of FM-I and AFM-I phases: the FM-I phase is dominant at low doping region, while the AFM-I phase (hatched peaks) gradually takes over the FM-I phase with increasing $x$. The present result is consistent with Ref. [6] and makes it clear that the planar FM phase is stable below $x \sim 0.40$. We also found that the diffraction pattern at $x=0.30$ is completely different from the other concentrations, and corresponds to the FM-II phase, in which spins are aligned ferromagnetically along the $c$-axis. The drastic change in the magnetic structure indicates that there exists a compositional phase boundary around $x=0.30-0.32$. To study the intermediate temperature phase, we have also meausured powder diffractions around $100-120 \mathrm{~K}$. As is consistent with Ref. [6], only the AFM-I phase remains for $x=0.39-0.50$, while no magnetic reflections are observed for $x=0.32-0.38$. As $x=0.30$, however, the low-temperature FM-II phase disappears and the AFMII phase appears at intermediate temperatures.

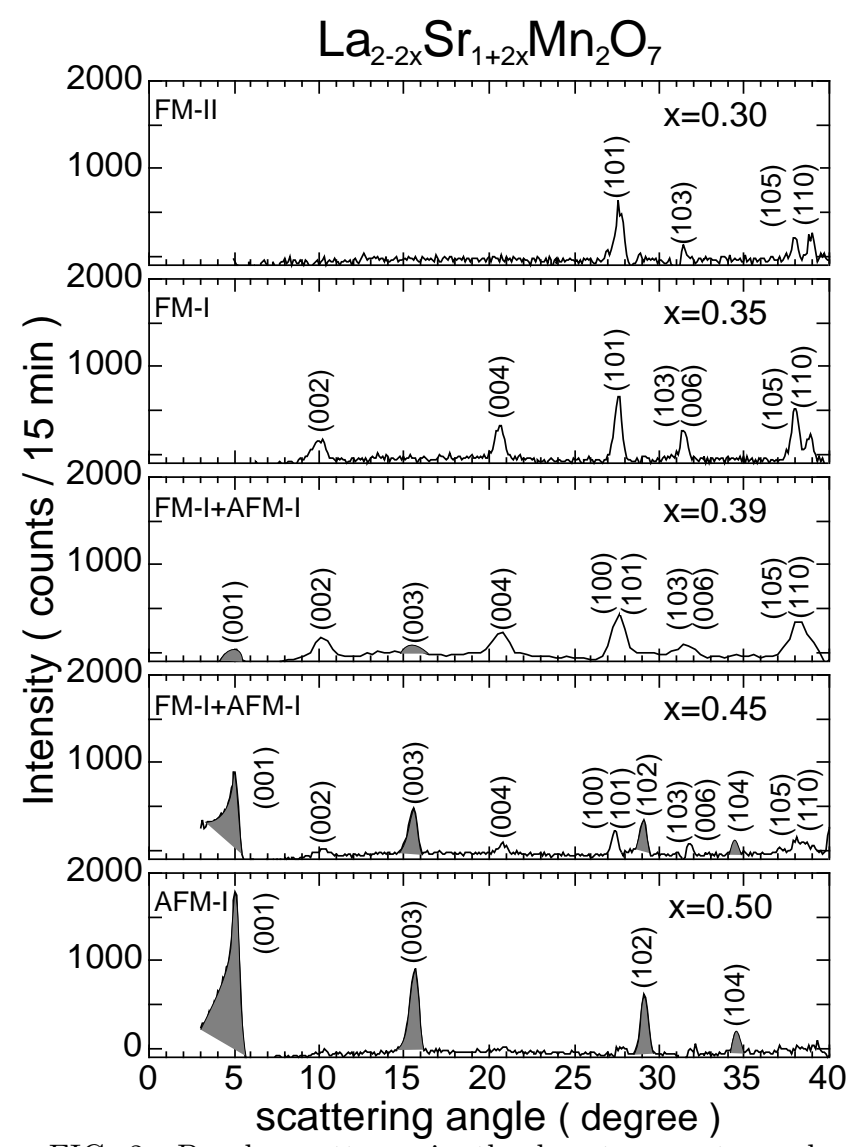

FIG. 2. Powder patterns in the low temperature phase $(\sim 10 \mathrm{~K})$ of $\mathrm{La}_{2-2 x} \mathrm{Sr}_{1+2 x} \mathrm{Mn}_{2} \mathrm{O}_{7}(0.30 \leq x \leq 0.50)$. The data taken at room temperature is subtracted from each profile as background. Hatched peaks correspond to AFM reflections, and the others correspond to FM reflections.

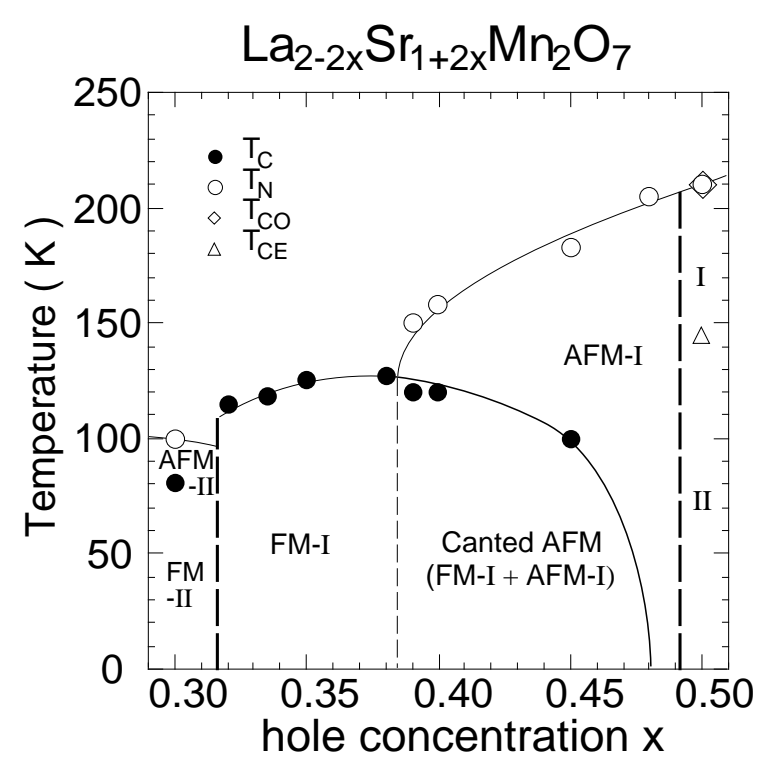


FIG. 3. Magnetic phase diagram of $\mathrm{La}_{2-2 x} \mathrm{Sr}_{1+2 x} \mathrm{Mn}_{2} \mathrm{O}_{7}$ $(0.30 \leq x \leq 0.50)$. AFM-I (AFM-II) indicates the planar A-type AFM structure with FM (AFM) intra-bilayer coupling and FM (AFM) inter-bilayer coupling. FM-I and FM-II stand for the FM structures with spin within the $a b$ plane and along the $c$-axis, respectively. (See Fig. 1) As for $x=0.50$, only AFM-I exists in the phase I, while AFM-I and CE-type AFM coexist in the phase II.

We also measured the temperature dependence of typical magnetic reflections to complete the magnetic phase diagram of LSMO327, as shown in Fig. 3. As for $0.32 \leq x \leq 0.48$, there is essentially one low-temperature magnetic phase, i.e., a planar canted AFM, in which the canting angle is zero below $x=0.38$, becomes finite at $x=0.39$ then gradually increases with increasing $x$, and finally reaches $180^{\circ}$ at $x=0.48$. Note that the intermediated AFM-I phase appears at $x=0.39$, where the canting angle in the low-temperature phase becomes finite. The magnetic phase becomes more complicated at $x=0.50$ because of the charge ordering. [9.10 Since superlattice peaks due to the charge ordering and CE-type AFM ordering are not confirmed in the present powder diffraction study because those peaks are very weak, we refer to the results of Ref. [10] in our phase diagram.

The magnetic phases for $x=0.30$ are completely different from the other concentrations. With decreasing temperature, it becomes the planar AFM-II phase at $\sim 100 \mathrm{~K}$ and then changes to the uniaxial FM-II phase at $70 \mathrm{~K}$. Perring et al. [8] also conclude that the low-temperature phase is AFM with spins aligning along the $c$-axis, which is inconsistent with the FM-II structure in the present study, though the change of the easy-axis direction from planar to uniaxial at intermediate temperature is similar. Note that there exists a compositional phase boundary at $x=0.30-0.32$, which is consistent with difficulties in growing a crystal. More detailed study, particularly with a good single crystal, is required to completely clarify the magnetic structure around $x=0.30$.

Recent studies indicate that there is a close relation between the magnetism and structure of LSMO327, particularly through the $\mathrm{Mn} e_{g}$ orbital degree of freedom. [6, 12, 13, 14, 15, It is thus necessary to study the hole concentration dependence of structure in detail and compare with the magnetic phase diagram we have established in the present study.

[1] A. Urushibara, Y. Moritomo, T. Arima, A. Asamitsu, G. Kido and Y. Tokura, Phys. Rev. B 5111103 (1995).

[2] A.J. Millis, P. B. Littlewood and B. I. Shraiman, Phys. Rev. Lett. 745144 (1995).

[3] Y. Moritomo, Y. Tomioka, A. Asamitsu and Y. Tokura, Phys. Rev. B 513297 (1995).

[4] Y. Moritomo, Y. Tomioka, A. Asamitsu, Y. Tokura and Y. Matsui, Nature (London) 380141 (1996).
[5] J. F. Mitchell, D. N. Argyriou, J. D. Jorgensen, D. G. Hinks, C. D. Potter and S. D. Bader, Phys. Rev. B 5163 (1997).

[6] K. Hirota, Y. Moritomo, H. Fujioka, M. Kubota, H. Yoshizawa and Y. Endoh, J. Phys. Soc. Jpn. 673380 (1998).

[7] T. Kimura, A. Asamitsu, Y. Tomioka and Y. Tokura, Phys. Rev. Lett. 793720 (1997).

[8] T. G. Perring, G. Aeppli, T. Kimura, Y. Tokura and M. A. Adams (unpublished).

[9] J. Q. Li, Y. Matsui, T. Kimura and Y. Tokura, Phys. Rev. B 57 R3205 (1998).

[10] M.Kubota, H. Yoshizawa, H. Fujioka, K. Hirota, Y. Moritomo and Y. Endoh (unpublished).

[11] K. Ohoyama, T. Kanouchi, K. Nemoto, M. Ohashi, T. Kajitani and Y. Yamaguchi, Jpn. J. Appl. Phys. 373319 (1998).

[12] I. Solovyev, N. Hamada and K. Terakura, Phys. Rev. Lett. 764825 (1996).

[13] S. Ishihara, J. Inoue and S. Maekawa, Physica C, 263, 130 (1996).

[14] D.N.Argyriou, J. F. Mitchell, C. D. Potter, S. D. Bader, R. Kleb and J. D. Jorgensen, Phys. Rev. B 55 R11965 (1997).

[15] Y. Moritomo, Y. Maruyama, T. Akimoto and A. Nakamura, J. Phys. Soc. Jpn. 67405 (1998). 